\title{
Anthropomorphic Networks as Representatives of Global Consciousness
}

\author{
Sergii Yahodzinskyi ${ }^{1}$ \\ Doctor of Philosophical Sciences, Associate Professor, National Aviation University \\ (Kyiv, Ukraine) \\ E-mail: sophist@nau.edu.ua \\ ORCID: 0000-0001-8755-2235
}

There has been analyzed a phenomenon of global consciousness, and its cultural and historical, civilizational dimensions have been substantiated. There has been demonstrated that the concept of planetary consciousness, global thinking, noosphere was described for the first time in the philosophy of cosmism. However, in modern conditions ideas of representatives of the naturalistic philosophical direction of cosmism have not lost their heuristic potential. They can be reconsidered in a new fashion within the context of emerging anthropomorphic (human dimension) networks. There has been proved that global consciousness is a component of the social and cultural potential of global information networks defining vectors to prospects of humanity progress in the $21^{\text {st }}$ century. Relying on methodology of the structural and functional analysis, the author arrives at a conclusion about global networks obtaining the status of representatives of global consciousness. This is the area of networks where all relevant information is concentrated - from statistical data to scientific and technical information. Access to these data is limited by human abilities and is realized in the form of discrete requests with using heuristic algorithms of information procession. A suggestion is introduced considering the fact that modern society being a self-organized system seeks to gain stable condition. Anthropomorphic networks are means of decreasing social entropy, which is growing as a result of any kind of human intervention into social processes. Thus, for the first time a human is challenged by their intellect, ability to create, discover and control.

Keywords: global consciousness, information society, network society, anthropomorphic information networks, philosophy of cosmism, discursive practice, sociocultural practice.

Received 15 April 2017; accepted 20 May 2017

Philosophy and Cosmology, Volume 20, 2018: 146-153

DOI: $10.29202 /$ phil-cosm/20/14

\section{Introduction}

The founder of the concept of collective memory, Moris Halbwachs already in the first half of the $20^{\text {th }}$ century introduced a suggestion that society is something more than a community of people, their beliefs, aspirations, interests and institutions developed on this basis. However, social synergy is driven only when social solidarity is realised in harmony of

(C) Yahodzinskyi, Sergii, 2018 
thinking, feelings and actions. The aim of such changes is development of a method used by "collective mind... to give human consciousness access to everything this mind realises itself in: to feelings, behaviour and priorities of different social groups" [Halbvachs, 2000: 168]. This requires society to take different collective decisions on existing and new sociocultural techniques. Having been tested in the social field, they are to become a usual social practice aimed at consolidating trust and efforts on extending medium mastered by humankind.

Within this context, sociocultural practice is in line with discursive practice. Thus, notion of discursive practices introduced into scientific circulation by Mishel Fuko is being widely used in modern social and humanitarian research. Considering the fact that discursive practices are rules set by a certain historical epoch, there is a temptation to subordinate sociocultural practices to them as well. However, according to our opinion, interpretation of sociocultural practices as a subtype of discursive practices is inadmissible. It leads to the state when we have to recognize anthropomorphic networks to be the resources of global consciousness, the discursivity of which is based on the stage of their construction.

To substantiate this point, let us consider the functional components of discursive practice. Each of them, according to Mishel Fuko, undergoes three stages of development: derivation (composition of essence); mutation (implementation of interdiscursive dependencies); redistribution (establishment of a new hierarchical order). Though sociocultural practices are also aimed at search for new kinds of society's life activity, their main function is different. It lies in expanding these kinds for the maximum level of society's sociocultural potential realisation in such conditions. By analogy, discursive practice is a metaphysical, while sociocultural is an ontological level of rule legitimization and social reality organization.

Resource dilatability of exclusively discursive practices was noticed by Mishel Fuko himself. In one of his works, he writes, 'Since the last century, great battles questioning the general system of power don't happen anymore... No one expects from the emperor or the kingdom the last days any more nor even just the return of their original rights... what they really stand for and hold as an aim is life interpreted in terms of fundamental needs, a specific essence of a human, realisation of their vitalities', fullness of the possible' [Fuko, 1996: 250]. Such conclusion converts easily into postmodern nihilism, games, simulacra, destruction.

The aim of existence of humankind dissolved in artificial discourses (ways of power retention) disorients social groups and society in general. Absence of common business, new horizons and real, not virtual prospects is able to retrieve social evolution by destroying institutions and negating social consciousness. Once this collision was discovered by representatives of the cultural and civilizational approach to periodization of history, but they did not manage to suggest the way of its elimination. Removal of the contradiction between culture and civilization, in our opinion, is the task for sociocultural practices aimed at searching for a medium, a representative of global consciousness.

\section{Methodology: Planetary Consciousness as a Theoretical Construct}

This task becomes of special relevancy in informational society, which was the first to give a status of the conceptual social project to the issue of providing humankind unity. Successful implementation of the range of innovative solutions on the cusp of the $20^{\text {th }}-21^{\text {st }}$ centuries accelerated integration processes in the spheres of economy, culture, law, education and military sector. Considering those, we consider it relevant and necessary to introduce to the social and philosophical notion and category base notions "global thinking", "global consciousness", "planetary personality", "collective mind" etc. These notions are to describe social medium as a consolidated one, able to overcome inner conflicts, racial, religious and 
other forms of hatred. In other words, modern society possesses resources for representing global consciousness as sociocultural practice.

In this paper, we use the term within the context of the social and constructivist philosophical approach and mean by it a combination of ideas, principles, theories that can be accepted by society as standardised for providing social progress and ensuring survival of humankind. Subject to substantiation of the need of objectification, sociocultural constructs receive ontological equality with structural components of social reality. Morale, money, state power, classes, ranks, titles etc. are examples of constructs and civil practices implemented into the social medium [Reznik, 2011: 14-33]. Their importance, apart from radical social and philosophical interpretations, is beyond doubt, and thus in most countries of the world they have served as a basis for social medium formation.

Every historical epoch produced new constructs on the basis of its cultural and civilizational coordinates and transformed the existing ones. At the same time, reasons for introducing the notion of global consciousness to the level of sociocultural construct are still not answered. Subject matter and role of the defined construct in realisation of information era social potential cause as many discussions among scientists as the previous issue.

In our opinion, there are several reasons of such sceptical attitude. First, modern civilizational challenges are so unpredictable and global that the idea of their solution by imposing another theoretical construct on social consciousness seems to be illusive. Second, postmodern experience of overcoming narratives has established lack of trust to them and complicated their development and implementation. Third, ethical orientation of most sociocultural constructs reduces social trust to them in the conditions of intensifying technologies of public opinion manipulation.

Taking that into account, the only way of involving innovative, cognitive and cultural potential of a certain construct we see presupposes defining its historical and cultural, cultural and civilizational sources.

When searching for conceptual analogues of the global consciousness construct, researchers have continuously addressed philosophy and worldview legacy of cosmism representatives (Vladimir Vernadskyi, Olexandr Chyzhevskyi, Kostiantin Tsiolkovskyi, Mikola Umov and others). Nowadays, being a scientific and philosophical system, cosmism develops the vision of macrocosm integrity and interconnection between the Universe and social life processes [Krichevsky, 2017: 52-54]. Cosmism representatives hold the opinion that space is a house for humankind to understand and acquire. Social and philosophical, and anthropological essence of cosmism can be described as follows: Humankind is a phenomenon of not only planetary, but also space origin, and thus it will become a factor of changing not only earth, but also space nature.

\section{Global Consciousness within the Context of Modern Worldview}

"Cosmic philosophy" raised the issue of forming a human with developed intellect, high moral qualities and culture of thinking, a human that is not only to master secrets of the Universe, but also to use the knowledge obtained for this mastering. Conclusions of cosmism representatives correlate with up-to-date naturalistic theories and experience of the humanities. Special attention should be paid to the line connecting cosmism and synergetic. Laws of non-equilibrium thermodynamics reflecting non-linear, cooperative etc. properties of open self-regulating systems highlight correctness of the worldview orientation defined by cosmists. Thus, conceptual framework of the philosophy of cosmism has preserved its relevancy and is constantly getting specified and expanding in modern papers. 
According to Oleg Bazaluk, 'the worldview of a future human is to be based on their understanding that their life is a part of life of civilization, extent of their self-realisation in life, scale and productivity of civilization activity' [Bazaluk, 2009: 4]. Nevertheless, at the level of individual consciousness, such warnings are considered as no more than metaphors and sometimes as elements of political struggle. Therefore, considering such statements relevant, justified, a human does not take sufficiently substantial actions for their realisation.

Moreover, the issue is not only in lack of social trust to such information media. The inhibiting factor is also absence of triggering mechanisms of sociocultural practices, which would fulfil and demonstrate advantages of collective care for future of civilization. One might accuse such statements of populism and unviability in actual conditions of social reality.

However, history and the present are encouraging more and more to admit objectivity of social consciousness evolution. Nikolay Berdyaev argued this process as follows: 'During its historical destiny, mankind goes through different stages, and their destiny is always tragic. At the beginning, human was a nature's slave, and they began heroic fight for their survival and independence. They created culture, states, national groups, classes. However, they became slaves of state, nationality, classes. "Today they start a new period' [Berdyaev, 1933: 36]. Similar to cosmism representatives, he developed his model of a new social order and described the way humankind moves there through moral Christian improvement.

Reality of the $20^{\text {th }}$ century civilization progression discovered a social utopian character of most suggested theories by bringing them to the complex of vectors, basic ideas and principles. Akop P. Nazaretyan asks: "How far can the development in morals and concomitant aggression-restrictors go?" [Nazaretyan, 2015: 96]. These are the latter ones that serve as a basis for renovated concepts of transforming social consciousness to global, planetary level. Denying the need in it means deepening those crisis phenomena, which are progressively devouring countries of the world. At the same time, Igor Kravchenko explains that we have passed the period of utopian expectations always preceding the social crisis [Kravchenko, 2004: 42-43]. Desiring to reconstruct the existing order, humankind aggravates cultural and civilizational contradictions, brings economy, culture, politics, law to recession. With time, it will inevitably negate sociocultural expectations to social nihilism and apathy.

Now it is time to admit that mankind has come up to another verge defining new visions about size of the accessible space, specific features of social relations, and legal and moral norms as well, outlining value guiding lines and sociocultural prospects. Denial of it is certainly a harmful social practice. Sergey Katrechko has the same position and calls transition to using information technologies a precondition of radical changes in consciousness, saying that "this enables us to speak not only about its transformations, but also about overcoming individual consciousness by over-individual structures' [Katrechko, 2004: 65]. We are certain that the very idea of global consciousness (with its modern content) brings the third dimension to social medium physically expanding it.

However, for moving in three directions - civilizational, cultural and noospheric, humankind must adjust all forms of social consciousness to global perspective. In one of his last works, the famous specialist in planetary thinking and collective mind, academician Nikita Moiseev was cautioning against superficial, premature conclusions concerning universal character of the idea about noosphere. Departure of social consciousness from conventional canons, norms and regulations itself provokes appearance of bifurcations known for their ability to deprive the system of 'memory' [Moiseev, 2000: 124]. For social system, it means losing its stable conditions. Partially these processes are already being observed in the form 
of neglecting humanitarian component in education, absence of institutes of moral, aesthetic, axiological social life support etc. Thus, we agree with Nikita Moiseev conclusion that 'one cannot claim that coming of noosphere is as much inevitable as the one of ecological crisis' [Moiseev, 2000: 130]. Nevertheless, there are no alternative ways of social progression.

\section{Concept of Global Consciousness in the Network Society}

One might assume that innovations and network technologies would slightly reduce civilizational tension by offering, though virtual, expanding of social medium under the conditions of social time curtailing. In particular, this way was used by authors of the collective monograph who gave to virtual networks the status of special reality affecting all aspects of the modern culture [Vliyanie Interneta, 2004]. Dmitrii Ivanov was also that optimistic about prospects of virtualization and considered it more heuristic than postmodernism. Therefore, summarizes the scholar, 'relevancy of focusing on virtualization at the beginning of the $21^{\text {st }}$ century will be growing and getting more obvious... at the same time, globalization paradigm which is now most fashionable will struggle in facing new tendencies' [Ivanov, 2000: 96]. However, even as a draft version, the concept of visualization might become a model for social changes at the turn of the 20-21 centuries. If researchers are right with their suggestion, global consciousness, in our opinion, should have resulted from the introduction of network social architectonics where sophisticated system of relations is caused by nonlinear differentiation of its elements. After translating it into the language of systems, we get a law of direct dependence of social actions over the whole social space if it is globalized.

Skilfully organized control over information flows, timely (even automatic) reaction on their divergence, detection of social bifurcation points, replacement of real objects, events, phenomena, causes with their simulacra constitute a virtual superstructure over the social system. Its advantages include adjustment to scale, universality, totality, physical indestructibility of resources and their reproduction in accordance with the speed of scientific and technological progress.

Therefore, today information networks define social metrics and seem more distinctly to be sufficient tools for transforming social reality into the virtual plane. Consequently, social space does not expand - as creators of digital revolution wanted it to happen - but narrows with every technological or socially humanitarian innovation. That is the reason why at the beginning of the $21^{\text {st }}$ century researchers keep trying to discover modern contexts and challenges accompanying global consciousness formation.

Particularly, Halina Nomerovskaia chose global consciousness as a special subject of her research [Nomerovskaia, 2006]. Considering historical character of the correspondent phenomenon, she sees its potential in providing sustainable civilization development. This is the basis for her conclusion that global consciousness is a component of globalists' and has to be responsible for the process of personality socialization in globalised social space. Morality, environmental friendliness, responsibility are to become attributes of a future human. Though one can agree with most conclusions offered by Halina Nomerovskaya, yet, in our opinion, generally they are directed not at description of real society transition to global level of its self-consciousness, but at philosophical and historical reflexion over this issue within the context of integrative and globalization processes of information society.

Evgeniy Prilutskiy interprets the problem of global consciousness formation in different way. Being a historian, he focused on genesis of this notion and followed its evolution from stating the issue of global thinking at the end of the $19^{\text {th }}$ - beginning of the $20^{\text {th }}$ century through vision about planetary consciousness and collective mind (Nikita Moiseev) to 
discovering influence of the Internet on all forms of social consciousness [Prilutskiy, 2013]. Though the vector of analysis he chose is illegally deflected to ecologism, we appreciate the idea about indiscrete aggression of global consciousness system against human and their psychophysiological abilities.

A human, having employed informational networks, is becoming today their soft touch. Still, decision making in situations requiring simultaneous consideration of a great number of input parameters and analysis of their mutual correlation is excessive even for the most gifted. In the middle of the $20^{\text {th }}$ century, there were few such tasks, but they also were not able to work for long time without assistance of operators. At the beginning of the $21^{\text {st }}$ century, distributed computation systems and non-linear algorithm systems have become general, and they mostly do not require active assistance from human in their operation. In the conditions of global information, networks being increasingly separated from the social, all possible parameters of society life activity get collected in the network.

Considering the above stated, one could draw a conclusion that human faces the verge, which is caused by their properties as a natural body. To overcome this barrier, society must get consolidated and adopt mutual norms, principles, laws of social life and, by general consent regarding that, move to master new space horizons. Such conclusion, though with elements of social utopism, is still sufficiently attractive from the point of view of representatives of social-political and humanitarian sciences. Without denying existing disadvantages of social reality, it shows the way of its further renovation, improvement, evolution, adaptation etc.

\section{Network as a Representative of Global Consciousness}

Nevertheless, such point of view has the right to exist only under one condition, which has never been disputed, by any thinker, scholar, and religious figure during the whole history of civilization. It has always been postulated that human is the only bearer of consciousness, and, correspondingly, humankind is the only bearer of global consciousness.

Getting out of these limitations, we can suppose appearance of anthropomorphic networks, which will 'impersonate' global consciousness. Such supposition seems like science fiction, but for several years already specialists from the European Organization for Nuclear Research (CERN) have been involving resources of both individual computers and separated networks while conducting hypercomplex calculations of hadron collider operation. Even processed data constitute so considerable amount of information that it cannot be recorded operatively on any standard medium, therefore, it exists in virtual network form only. It won't be an exaggeration to claim that this very network prototype, through using capacity of all resources available and correlating on-line legacy of hundreds of scientific groups from all over the world, accumulates a more complete image of macrocosm than the vision scholars have. Practically, a researcher when doing theoretical calculations has to make inquiries to the network clearly specifying what he is interested in. Herewith, complete amount of data in human consciousness is not available.

Technocrats are connecting their dreams about the so-called quantum computer with these realities more and more visibly. According to predictions, its computation capacity will enable to calculate and discover things, which are impossible even for up-to-date mathematical, sociological, political, ecological and other concepts. Immediate procession of Internet publications, forums, newspapers, press-releases, documents, monographs, articles, thoughts expressed in social networks and other available information will enable the quantum computer to evaluate within the accuracy of one day possibility of political, cultural, economic, military, educational events in the world for three years in advance. 
Moreover, every combination of events will be introduced as a completed configuration of the global social space.

\section{Conclusion}

In this article we have not covered the analysis of social-cultural consequences of implementing these innovative technologies; furthermore, a substantial number of general audience and futurological literature and tens of films are dedicated to this issue. The latter illustrates different variants of events after technological singularity establishment - a moment in history after which human won't be able to appreciate, comprehend and keep up independently with the rates of scientific and technological progress. It is not improbable that this direction of social development is natural and inevitable, and our concern is only a form of fear of changes. In fact, a society, like any other self-organized system, longs for stability and, by all means, will avoid destruction of well-established connections and relations. However, today human for the first time faces the challenge of their intellect, their ability to create, control, perceive. Is society ready to face global problems, to delegate part of management to virtual network? That is the question we have to deal with in the nearest decades.

Summing up the results of our research, we would like to direct attention of all social subjects to threats humankind has faced on the cusp of the $20^{\text {th }}-21^{\text {st }}$ centuries. We are far from apocalyptic predictions and sci-fi stories about future. At the same time, as it was described above, process of society incoming to the horizon of bifurcation point events in both technological and social-political and humanitarian spheres has begun. Thus, without development of social-cultural practices directed at forming global consciousness, global responsibility, global assessment of dependencies and balances, society risks to lose the image it has had for several millennia.

\section{[Qd] References}

Bazaluk, Oleg. Existence of World: a Modern Model "Evolving Matter". Philosophy \& Cosmology, 2009: 3-36.

Berdyaev, Nikolay. Human and Machine (Problem of Sociology and Metaphysics of Technology). Put, Vol. 33, 1933: 3-38.

Foucault, Michel. The Will to Truth: the Other Side of Knowledge, Power and Sexuality. Works of Different Years. Moscow: Kastal, 1996.

Halbvachs, Moris. Social Classes and Morphology. Moscow: Institute of Experimental Sociology, 2000.

Ivanov, Dmitriy. Virtualization of Society. St. Petersburg: Peterburgskoe Vostokovedenie, 2000.

Kamenskaya, Tatyana. Virtualization and Devirtualization of Social Reality. Methodology, Theory and Practice of Social Analysis of Modern Society. Vol. 16, 2010: 105-109.

Katrechko, Sergey. Internet and Consciousness: To the Concept of Virtual Human. Influence of the Internet on Consciousness and Knowledge Structure. Moscow: IFRAN, 2004: 5772.

Kravchenko, Igor. Politics and Consciousness. Moscow: IFRAN, 2004.

Krichevsky, Sergey. Cosmic Humanity: Utopia, Realities, Prospects. Future Human Image.7, 2017: 50-70.

Moiseev, Nikita. System Organization of Biosphere and the Concept of Coevolution. Social Sciences and the Present Time. Vol. 2, 2000: 123-130. 
Nazaretyan, P. Akop. Mega-History and the 21st Century Singularity Puzzle. Philosophy \& Cosmology. Vol. 15, 2015: 84-98.

Nomerovskaya, Halina. Formation of Global Consciousness: Philosophical Analysis: diss. ... cand. philos. sciences: 09.00.08 - Philosophy of Science and Technology. Moscow, 2006.

Prilutskiy, Evgeniy. Global and Planetary Consciousness (Notions, Historical Development, Subject). Samolit, 2013. http://samolit.com/books/5684.

Radin, Dean. The Conscious Universe: The Scientific Truth of Psychic Phenomena. HarperCollins, 1997.

Reznik, Oleksandr. Civil Practices in Transitional Society: Factors, Subjects, Methods of Realisation. Kyiv: Institute of Sociology of the NAS of Ukraine, 2011.

Vernadskiy, Vladimir. Living Matter and Biosphere. Moscow: Nauka, 1994.

Influence of the Internet on Consciousness and Knowledge Structure. Moscow: IFRAN, 2004.

Voronkova, Valentina. The Formation of the Concept of Noosphere Development of Modern Society in the Conditions of Information Society. Philosophy \& Cosmology. Vol. 16, 2016: 179-189. 